\title{
CORRECTION TO OUR PAPER “ON HEREDITARY RADICALS"
}

\author{
by \\ F. SZÁSZ and R. WIEGANDT (Budapest)
}

The authors are grateful to Dr. B. J. GARDNER for the following observations.

In our paper "On hereditary radicals", Period. Math. Hungar. 3 (1973), 235-241, the statement of Corollary 2 is not that what we have proved. The correct version of Corollary 2 is the following:

If the radical $\mathbf{R}$ is non-hereditary, then there are hereditary radicals $\mathbf{P}$ and $\mathbf{Q}$ such that neither $[\mathbf{P}, \mathbf{R}]$ nor $[\mathbf{R}, \mathbf{Q}]$ contains hereditary radicals except $\mathbf{P}$ and $\mathbf{Q}$, respectively.

As it has been pointed out by Dr. Gardner, the whole class $[\mathbf{P}, \mathbf{Q}]$ may contain other hereditary radicals, too. For instance, let $\mathbf{R}$ be the radical class of rings on groups of the form $A_{p} \oplus A_{q}$ where $A_{p}$ is a direct sum of copies of $C\left(p^{\infty}\right), A_{q}$ is a direct sum of copies of $C\left(q^{\infty}\right)$, and $p, q$ are different primes. Then the maximal hereditary radical class $\mathbf{H}_{R}$ contained in $\mathbf{R}$, is the class $\mathbf{0}$ consisting of the ring 0 , while the minimal hereditary radical $\overline{\mathbf{R}}$ containing the radical $\mathbf{R}$, is the class $\mathbf{B}(p, q)$ of prime radical rings on direct sums of $p$-groups and $q$-groups. If $\mathbf{B}(p)$ denotes the class of prime radical $p$-rings, then $\mathbf{B}(p)$ is hereditary and $\mathbf{B}(p) \in[\mathbf{0}, \mathbf{B}(p, q)]$ holds.

(Received May 6, 1973)

MTA MATEMATIKAI KUTATÓ INTÉZETE

H-1053 BUDAPEST

REÁLTANODA V. 13-15.

HUNGARY 